\title{
Ulrich Ermann, Ernst Langthaler, Marianne Penker und Markus Schermer (2018): Agro-Food Studies. Eine Einführung. 260 Seiten. Wien, Köln, Weimar: UTB Verlag
}

\author{
Jana Rückert-John
}

(C) Der/die Autor(en) 2020

Mit ihrem Buch „Agro-Food Studies“ bringen der Humangeograf Ulrich Ermann, der Sozial- und Wirtschaftswissenschafter Peter Langthaler und die SoziologInnen Marianne Penker und Markus Schermer eine im angloamerikanischen Sprachraum bereits langjährig etablierte wissenschaftliche Debatte in den deutschsprachigen Raum. Mit den zwei zentralen Begriffen „Agro“ und „Food“ wird der Blick auf die gesamte Lebensmittelwertschöpfungskette gelenkt. Hiermit ist der Anspruch verbunden, die einzelnen Phasen sowie die damit verbundenen sozialen Praktiken von der Produktion der Nahrungsmittel bis hin zum Essen und Trinken systematisch zusammenzudenken und der strikten Trennung der Kettenglieder entgegenzuwirken. Denn die Ausdifferenzierung der Lebensmittelwertschöpfungsprozesse lässt sich als eine wesentliche Ursache für verschiedene Probleme im Agro-Food-Bereich identifizieren. Auch die Wissenschaft ist durch diese funktionale Differenzierung geprägt: Agrarwissenschaften stehen Ernährungswissenschaften gegenüber, naturwissenschaftliche Betrachtungen unterscheiden sich von sozial- und kulturwissenschaftlichen Perspektiven auf Produktion und Konsum. Ein vergleichbares Themengebiet, das diese notwendige Verbindung (wieder) in den Blick nimmt und die Disziplinen verknüpft und nicht trennt, gibt es im deutschen Sprachraum nicht, weshalb die AutorInnen sich für den englischsprachigen Titel entschieden haben. Agro-Food Studies bezeichnet deshalb keine einzelne Theorie oder Wissenschaftsdisziplin, sondern vielmehr eine spezifische Forschungsrichtung, die inter- und transdisziplinär arbeitet und eine kritische Gesellschaftsperspektive verfolgt. Aus Sicht der AutorInnen schließen Agro-Food Studies ,eine Lücke in den überwiegend betriebswirtschaftlich, naturwissenschaftlich und technisch orientierten Forschungseinrichtungen und Studiengängen im Agrar- und Ernährungsbereich“ (S. 11).

J. Rückert-John $(\bowtie)$

Hochschule Fulda, Leipziger Straße 123, 36037 Fulda, Deutschland

E-Mail: jana.rueckert-john@oe.hs-fulda.de 
Das Buch ist vor dem Hintergrund der genannten Zielsetzung entlang von Dichotomien konzipiert. Nicht nur der Titel des Buches greift mit dem Gegensatz zwischen Produktion und Konsum eine Zweiteilung auf, auch die zentralen Kapitel sind entlang von acht Wortpaaren konzipiert, die jeweils einen Gegensatz bilden: Tradition und Moderne, Globalisierung und Regionalisierung, Gesellschaft und Umwelt, Natur und Technik, Kopf und Bauch, Überfluss und Mangel, Verbindendes und Trennendes sowie Stabilität und Veränderung.

Die AutorInnen nutzen zunächst die dichotomen Denkmuster als etablierte und vertraute Unterscheidungen der Problemanalyse, um sie dann infrage zu stellen und alternative Sichtweisen aufzuzeigen (1). Denn ,Agro-Food Studies verstehen sich als ein alternatives Projekt, das Gegenentwürfe zu einer von der Gesellschaft abgehobenen Agrar- und Ernährungswissenschaft bereitstellt“" (S. 13). Die AutorInnen versuchen dichotome Denkmuster auch dadurch zu überwinden, indem sie Theorieansätze verwenden, die einen integrativen Blick jenseits von reduktionistischen Ansätzen erlauben, so zum Beispiel die transition theory für das Kapitel ,Stabilität und Veränderung“ (2). Zudem verfolgen die AutorInnen mit ihrem Buch einen konsequent interdisziplinären Anspruch, um die vorgestellten Dichotomien aufzulösen (3). Hierzu rezipieren sie überwiegend englischsprachige Literatur, die sie mit der deutschsprachigen Forschung verbinden und durch ihre wissenschaftlichen Interpretationen an- und bereichern. Ihnen ist es hierbei besonders wichtig, den Globalen Süden angemessen zu berücksichtigen und keine eurozentrische Sichtweise zu verfolgen.

Die acht zentralen Kapitel folgen einer einheitlichen Struktur. Sie beginnen mit einer kurzen Zusammenfassung, sodann folgt eine Darstellung des jeweiligen theoretischen Leitkonzepts, im Weiteren schließen sich detailliertere Ausführungen anhand empirischer und praktischer Beispiele an. Jedes Kapitel endet mit Kontroll- und Diskussionsfragen, die der Wiederholung und Vertiefung des Wissens dienen. In einem Glossar am Ende des Buches werden die zentralen Fachbegriffe kurz erläutert.

Ihr Werk verstehen die AutorInnen als eine interdisziplinäre Einführung in die Agro-Food Studie, für deren Lektüre es keines fachspezifischen Vorwissens bedarf, wenngleich viele Verweise auf einschlägige Fachdiskussionen und entsprechende Literatur gegeben werden. Die AutorInnen wenden sich mit ihrem Buch vor allem an Studierende, die am Beginn ihrer Beschäftigung mit Themen der Agrar-, Lebensmittel- und Ernährungsforschung stehen. Es eignet sich daher vor allem für den Einsatz im Grundlagenstudium der Agrar- und Ernährungswissenschaften, aber auch für spezielle Lehrveranstaltungen im Agro-Food-Bereich, wie der Soziologie, Kulturwissenschaften, Geografie, Ökonomie und Geschichte. Die AutorInnen verstehen ihr Buch jedoch nicht als ein klassisches Lehrbuch, das sich als Kompendium oder Nachschlagewerk eignet, um gesichertes Wissen zu Fragen der Lebensmittelproduktion und Ernährung bereitzustellen. Vielmehr soll es die LeserInnen „mit wichtigen Diskussionen und Grundperspektiven vertraut [machen] und [sie] an[regen], vermeintlich eindeutige Zusammenhänge auch anders zu sehen“ (S. 15). Diese Kompetenzen einer kritischen Gesellschaftsreflexion sind jedoch nicht nur bei Studierenden angezeigt, sondern sprechen auch andere Interessierte an Fragen der Agro-Food Studies aus den Bereichen Bildung, Wirtschaft, Politik und Zivilgesellschaft an. 
Das Buch überzeugt durch sein klar formuliertes Konzept und dem hieraus abgeleiteten logisch nachvollziehbaren Aufbau. Es eignet sich deshalb in besonderer Weise für die Lehre in den Agrar- und Ernährungswissenschaften. Das Buch bietet eine sehr gute Grundlage, um interdisziplinäre Perspektiven in diesen Wissenschaftsdisziplinen systematisch durch die Verbindung von Agro- und Food-Studies vorzustellen und kritisch zu diskutieren. Die Umsetzung des eigens gesteckten hohen konzeptionellen und methodischen Anspruchs, vorherrschende Gegensätze zu überwinden und kritische, gesellschaftsgestaltende Perspektiven zu eröffnen, ist den AutorInnen in hervorragender Weise gelungen.

Funding Open Access funding provided by Projekt DEAL.

Open Access Dieser Artikel wird unter der Creative Commons Namensnennung 4.0 International Lizenz veröffentlicht, welche die Nutzung, Vervielfältigung, Bearbeitung, Verbreitung und Wiedergabe in jeglichem Medium und Format erlaubt, sofern Sie den/die ursprünglichen Autor(en) und die Quelle ordnungsgemäß nennen, einen Link zur Creative Commons Lizenz beifügen und angeben, ob Änderungen vorgenommen wurden.

Die in diesem Artikel enthaltenen Bilder und sonstiges Drittmaterial unterliegen ebenfalls der genannten Creative Commons Lizenz, sofern sich aus der Abbildungslegende nichts anderes ergibt. Sofern das betreffende Material nicht unter der genannten Creative Commons Lizenz steht und die betreffende Handlung nicht nach gesetzlichen Vorschriften erlaubt ist, ist für die oben aufgeführten Weiterverwendungen des Materials die Einwilligung des jeweiligen Rechteinhabers einzuholen.

Weitere Details zur Lizenz entnehmen Sie bitte der Lizenzinformation auf http://creativecommons.org/ licenses/by/4.0/deed.de.

Jana Rückert-John Prof.in, Sozialwissenschaftlerin, Lehrstuhlinhaberin für „Soziologie des Essens“ an der Hochschule Fulda im Fachbereich Oecotrophologie, Vorstandsvorsitzende des Netzwerkes für Ernährungskultur, Forschungsschwerpunkte sind Wandel des Essalltags, Geschlechterforschung, Soziale Innovationen und Transformation, Agro-Food-Studies. 